Pacific Journal of Mathematic 


\title{
ON INDECOMPOSABLE INJECTIVES OVER ARTINIAN RINGS
}

\author{
Kent R. FulleR
}

For each primitive idempotent $f$ in a left artinian ring $R$, let $T(R f)$ denote the unique simple factor of $R f$ and let $E(T(R f))$ denote its injective hull. Then, identifying a module with its isomorphism class,

$$
f R \longleftrightarrow E(T(R f))
$$

provides a one-to-one correspondence between the finite sets of isomorphism classes of indecomposable injective left $R$ modules and indecomposable projective right $R$-modules. The importance of this correspondence is illustrated by the NagaoNakayama result that if $R$ is a finite-dimensional algebra over a field $K$ then, under the duality ()$^{*}=\operatorname{Hom}_{K}(\ldots, K)$ between the categories of finitely generated left and right $R$-modules, one has

$$
(f R)^{*} \cong E(T(R f)) \text {. }
$$

Thus the structure of an indecomposable injective module $E(T(R f))$ over such an algebra $R$ is completely determined by the indecomposable (projective) direct summand $f R$ of $R_{R}$. However, in the more general case when $R$ is a left artinian ring, very little is known about the structure of these indecomposable injective modules. In this paper we attempt to shed some light on the problem by showing that a large part of the above mentioned duality can be carried over to $f R$ and $E(T(R f))$ over a ring $R$ with minimum condition on left ideals.

The key to most of our results is annihilator relations between $f R$ and $E(T(R f))$ given by the lemma of $\S 1$.

If $S$ is the inverse $R$-endomorphism ring of the left $R$-module $E$ then $E$ is a left $R$-right $S$-bimodule. Moreover, $f R f$ is naturally isomorphic to the $R$-endomorphism ring of $f R$ and $f R$ is a left $f R f$-right $R$-bimodule. In $\S 2$ we show that, if $E=E(T(R f))$,

$$
(f r, x)=f r x, f r \in f R, x \in E \text {, }
$$

yields an orthogonal pairing between ${ }_{f R f} f R$ and $E_{S}$ in the sense of Tachikawa [16]. Because the $f R f$-submodules of $f R$ and the $S$-submodules of $E$ satisfy the annihilator conditions under this pairing, we are able to determine the simple submodules of the various Loewy factors of $E$ in terms of those of $f R$. As a consequence, a left artinian ring is $Q F-3$ if and only if the projective covers of its left injective modules are themselves injective.

Nakayama's original definition of quasi-Frobenious $(=Q F)$ rings 
amounts to saying that a ring $R$ with both minimum conditions is $Q F$ in case for each primitive idempotent $e$ in $R$ there is a primitive idempotent $f$ in $R$ with Socle $(R e) \cong T(R f)$ and Socle $(f R) \cong T(e R)$. In $\S 3$ we show that this condition is necessary and sufficient for any primitive left ideal $R$ e over a left artinian ring $R$ to be injective. Thus the injective projective left modules over such rings are characterized in terms of one-sided ideal structure. We also present generalizations of the annihilator and the duality characterizations of $Q F$ rings to injective left ideals. Using these results we see that a left artinian $Q F-3$ ring (i.e., $E\left({ }_{R} R\right)$ is projective) also has $E\left(R_{R}\right)$ projective.

In $\S 4$ we consider the Johnson-Wong [9] and Wu-Jans [19] notions of quasi-injectives and quasi-projectives. The annihilator conditions of $\S 2$ provide us with a natural one-to-one correspondence between the quasi-injective submodules of $E(T(R f))$ and the quasi-projective factors of $f R$. In the event that $R$ has both minimum conditions this correspondence yields one between the sets of isomorphism classes of left indecomposable quasi-injectives and right indecomposable quasiprojectives over $R$. Here we also show that a left artinian ring has an infinite number of nonisomorphic left indecomsable quasi-injectives if and only if its lattice of two-sided ideals is infinite; thus providing a dual result to a theorem of Wu and Jans [19].

In the concluding section we show that left quasi-projective quasiinjectives are taken to right quasi-injective quasi-projectives by the correspondence of $\S 4$. This result allows us to give several characterizations of generalized uniserial rings. Perhaps the most interesting of artinian ring is generalized these is that a left uniserial in case each of its left indecomposable projectives and each of its left indecomposable injectives has a unique composition series.

1. Notation and the key lemma. Our main concern shall be with injective modules over rings with minimum condition on left ideals (i.e., left artinian rings). However, to insure that certain of our results hold for right as well as for left modules, we are forced to operate within the framework of the usual right-left symmetric generalization of left artinian rings-semi-primary rings. A semiprimary ring $R$ is an associative ring with identity 1 and nilpotent Jacobson radical $N$ such that $R / N$ is semi-simple. We assume knowledge of the well known decomposition theorems for projective [4] and injective [11] modules over semi-primary and left artinian rings.

If $M$ is a left (right) $R$-module we write $S(M)$ for the socle of $M, T(M)=M / N M(T(M)=M / M N)$, and $E(M)$ for the injective hull [3] of $M$. If $J \subseteq R$ and $K \subseteq M$ we write

$$
\boldsymbol{l}_{J}(K)=\{j \in J \mid j K=0\}
$$


and

$$
\boldsymbol{r}_{K}(J)=k \in\{K \mid J k=0\}
$$

when $M$ is a left $R$-module. If $M$ is a right $R$-module $r_{J}(K)$ and $l_{K}(J)$ are defined in the obvious manner. Note that if $M$ is a left $R$-module then $S(M)=r_{M}(N)$; and that if $E$ is an indecomposable injective left $R$-module then $E \cong E(T(R f))$ where $f$ is a primitive idempotent in $R$ with the simple module $T(R f) \cong S(E)$.

As stated in the introduction, we intend to examine the relationship between $E(T(R f))$ and $f R$ for $f$ a primitive idempotent in $R$. However, for some of our results we shall need a slightly more general notion. If $f$ is any idempotent in $R$ then $f$ can be written.

$$
f=f_{11}+\cdots+f_{1 \mu(1)}+\cdots+f_{n 1}+\cdots+f_{n \mu(n)}
$$

where the $f_{i j}$ form an orthogonal set of primitive idempotents satisfying $f_{i j} R \cong f_{s t} R$ if and only if $i=s$. Letting $f_{i}=f_{i 1}, i=1, \cdots, n$, we say that $f_{1}, \cdots, f_{n}$ is a basic set of idempotents for $f$. If $E$ is an injective left $R$-module whose socle has a finite composition series then we can write

$$
E \cong E_{11} \oplus \cdots \oplus E_{1 \nu(1)} \oplus \cdots \oplus E_{m 1} \oplus \cdots \oplus E_{m \nu(m)}
$$

where the $E_{i j}$ are indecomposable injective modules with $E_{i j} \cong E_{s t}$ if and only if $i=s$. Letting $E_{i}=E_{i 1}, i=1, \cdots, m$ we call $E_{1}, \cdots, E_{m}$ a basic set of injectives for $E$. If $E\left(T\left(R f_{1}\right)\right), \cdots, E\left(T\left(R f_{n}\right)\right)$ form a basic set of injectives for $E$ we say that $E$ is paired to $f R$ or that $f R$ and $E$ form a pair.

Some of the most important examples of such pairs are

(a) If $R$ is a finite dimensional algebra over a field $K$ then the injective left $R$-module $(f R)^{*}=\operatorname{Hom}_{K}(f R, K)$ is paired to $f R$.

(b) If $E$ is indecomposable and $f$ is primitive then $f R$ and $E$ form a pair if and only if $E \cong E(T(R f)$ ).

(c) An injective module $E$ is paired to $R$ if and only if $S(E)$ is finitely generated and faithful as an $R / N$-module.

We are now in a position to state the lemma that is the foundation of this paper.

LEMma 1.1. Let $f R$ and $E$ form a pair over the semi-primary ring $R$. Then

(a) $\boldsymbol{r}_{E}(J)=\boldsymbol{r}_{E}(f J)$ and $\boldsymbol{l}_{f R}(K)=\boldsymbol{l}_{f R}(K E)$ for every left ideal $J$ and every right ideal $K$ in $R$;

(b) $r_{E}(f R)=0$ and $\boldsymbol{l}_{f R}(E)=0$;

(c) $r_{R}(f R)=l_{R}(E)$.

Proof. (a) Let $X=\boldsymbol{r}_{E}(f J), J \leqq{ }_{R} R$. It is apparent that $\boldsymbol{r}_{E}(J) \subseteq X$. 
Suppose $J X \neq 0$. Then, since $J X \leqq{ }_{R} E, J X \cap S(E) \neq 0$. This implies that $J X$ has a simple submodule that can be embedded in $T(R f)$ and hence that $f J X \neq 0$, a contradiction. Thus $\boldsymbol{r}_{E}(J)=\boldsymbol{r}_{E}(f J)$. Let $L=$ $\boldsymbol{l}_{R}(K E), K \leqq R_{R}$. Then $L \leqq{ }_{R} R$ and $f L=f R \cap L=\boldsymbol{l}_{f R}(K E) \supseteqq \boldsymbol{l}_{f R}(K)$. Suppose $f L K \neq 0$. Then there must exist left ideals $I^{\prime}<I \leqq L K$ with $I / I^{\prime}$ simple and isomorphic to a submodule of $T(R f)$. This gives a monomorphism $\varphi: I / I^{\prime} \rightarrow E$. If $\eta: I \rightarrow I / I^{\prime}$ is the natural epimorphism we have, by injectivity of $E$, that $\varphi \circ \eta(I)=I x$ for some $x \in E$. But then $L K E \supseteqq I x=\varphi \circ \eta(I) \neq 0$, contrary to the definition of $L$. This proves that $l_{f R}(K E)=l_{f R}(K)$.

(b) This part follows from (a) by taking $J=R$ and $K=R$.

(c) Let $A=\boldsymbol{r}_{R}(f R)$ and $B=l_{R}(E)$. Then, using (b), $f R B=f B=$ $\boldsymbol{l}_{f R}(E)=0$, so $B \cong A$. Also $A E \cong \boldsymbol{r}_{E}(f R)=0$, so $A \subseteq B$.

As an easy consequence of (1.1) we note that there is a natural one-to-one correspondence between the injective simple left $R$-modules and the projective simple right $R$-modules.

Proposition 1.2. Let $R$ be a semi-primary ring with primitive idempotent $f$. Then $T(R f)$ is injective if and only if $T(f R)$ is projective.

Proof. Since $f R$ is indecomposable, the exact sequence $0 \rightarrow f N \rightarrow$ $f R \rightarrow T(f R) \rightarrow 0$ splits if and only if $f N=0$. On the other hand, since $E(T(R f))$ is indecomposable, $E(T(R f)) \cong T(R f)$ if and only if $N \subseteq l_{R}(E(T(R f)))$. But the latter is true if and only if $f N=f R N=0$ by $(1.1, \mathrm{c})$.

Gerald Janusz recently informed us that he has been aware of (1.2) for some time. He also pointed out that using (1.2) is easy to show that $R$ is hereditary with $N^{2}=0$ if and only if every simple left $R$-module is either injective or projective.

2. The Loewy series of indecomposable injectives. If $M$ is a left module over a semi-primary ring $R$ and $p$ is the smallest positive integer satisfying $N^{p} M=0$, the upper Loewy series for $M$ is

$$
M>N M>\cdots>N^{p} M=0
$$

and the lower Loewy series for $M$ is

$$
0<\boldsymbol{r}_{M}(N)<\cdots<\boldsymbol{r}_{M}\left(N^{p}\right)=M
$$

(see [1, pp. 102-104]). Letting $N^{0}=R, N^{k-1} M / N^{k} M$ is the k-th upper Loewy factor of $M$ and $r_{M}\left(N^{k}\right) / r_{M}\left(N^{k-1}\right)$ is the $k$-th lower Loewy factor of $M$. Loewy series and factors for a right $R$-modules are defined in the obvious manner. 
Using well known properties of Loewy seres and the vector space dual one can show that if $R$ is a finite dimensional algebra, the $k$-th upper (lower) Loewy factor of $E(T(R f))=(f R)^{*}$ is isomorphic to $T\left(R e_{1}\right) \oplus \cdots \oplus T\left(R e_{m}\right)$ where $e_{1}, \cdots, e_{m}$ is a finite indexed set of primitive idempotents such that the $k$-th lower (upper) Loewy factor of $f R$ is isomorphic to $T\left(e_{1} R\right) \oplus \cdots \oplus T\left(e_{m} R\right)$. In this section we shall prove a related, though admittedly weaker, result for left artinian rings. For future use, we continue to state the lemmas in a setting more general than necessary for our immediate goal.

LEMma 2.1. If $f R$ and $E$ form a pair over the semiprimary ring $R$ then, for each left $R$-module $M$, restriction to $f M$ gives an abelian group isomorphism

$$
\operatorname{Hom}_{R}(M, E) \cong \operatorname{Hom}_{f R f}(f M, f E) .
$$

Moreover,

(a) the map \& defined via

$$
[\psi(s)](f x)=s(f x), s \in \operatorname{Hom}_{R}(E, E), f x \in f E,
$$

is a ring isomorphism from $\operatorname{Hom}_{R}(E, E)$ onto $\operatorname{Hom}_{f R f}(f E, f E)$;

(b) if $J^{\prime} \subseteq J$ are two-sided ideals in $R$ then

$$
\boldsymbol{r}_{E}\left(J^{\prime}\right) / \boldsymbol{r}_{E}(J) \cong \operatorname{Hom}_{f R f}\left(f J / f J^{\prime}, f E\right)
$$

as left $R$-modules.

Proof. Let $R, f R$ and $E$ be as in the hypothesis. Let $M$ be a left $R$-module. If $\alpha \in \operatorname{Hom}_{R}(M, E)$ then, for all $r \in R, m \in M$,

$$
\alpha(f r f m)=f r f \cdot \alpha(f m) \in f E .
$$

Thus $\left.\alpha\right|_{f M} \in \operatorname{Hom}_{f R f}(f M, f E)$ and restriction to $f M$ is an additive map from $\operatorname{Hom}_{R}(E, M)$ into $\operatorname{Hom}_{f R f}(f M, f E)$. If $0=\alpha(f M)=f R \alpha(M)$ then $\alpha$ is the zero map by $(1.1, \mathrm{~b})$. Let $\delta \in \operatorname{Hom}_{f R f}(f M, f E)$. If $r_{i} \in R$, $m_{i} \in M$ with $\sum r_{i} f m_{i}=0$, then for each $f r \in f R$,

$$
\begin{aligned}
f r\left(\sum r_{i} \delta\left(f m_{i}\right)\right) & =\sum f r r_{i} \delta\left(f m_{i}\right) \\
& =\delta\left(f r\left(\sum r_{i} f m_{i}\right)\right) \\
& =\delta(0)=0 .
\end{aligned}
$$

So $\sum r_{i} \delta\left(f m_{i}\right) \in r_{E}(f R)=0$ by $(1.1, b)$. Thus there exists an $R$-homomorphism $\bar{\delta} ; R f M \rightarrow E$ defined via

$$
\bar{\delta}\left(\sum r_{i} f m_{i}\right)=\sum r_{i} \delta\left(f m_{i}\right) .
$$

Using the injectivity of $E$, let $\hat{\delta}: M \rightarrow E$ extend $\bar{\delta}$. Then $\hat{\delta}(\mathrm{fm})=$ $\delta(f m)$ for all $f m \in f M$ and we have shown that restriction to $f M$ 
provides the desired isomorphism $\operatorname{Hom}_{R}(M, E) \cong \operatorname{Hom}_{f R f}(f M, f E)$.

To see that (a) holds take $M=E$.

For (b) note that, since $E$ is injective,

$$
\left[\rho\left(x+r_{E}(J)\right)\right]\left(j+J^{\prime}\right)=j x, x \in r_{E}\left(J^{\prime}\right), j \in J
$$

defines an isomorphism

$$
\rho: r_{E}\left(J^{\prime}\right) / r_{E}(J) \rightarrow \operatorname{Hom}_{R}\left(J / J^{\prime}, E\right) .
$$

But we know that

$$
\operatorname{Hom}_{R}\left(J / J^{\prime}, E\right) \cong \operatorname{Hom}_{f R f}\left(f J / f J^{\prime}, f E\right) .
$$

It is routine to check that these isomorphisms commute with left multiplication by elements of $R$.

According to $[7$, p. 48 , Proposition 1] $f N f$ is the radical of the ring $f R f$. Hence if $R$ is semi-primary (or artinian) so is $f R f$; and if $f_{1}, \cdots, f_{n}$ is a basic set of idempotents for $f$ then $T\left(f R f_{i}\right)=f R f_{i} / f N f_{i}$, $i=1, \cdots, n$, are all the (non-isomorphic) simple left $f R f$-modules.

LEMMA 2.2. If $f R$ and $E$ are as in (2.1) then the right $f R f$ module $f R f$ and the left $f R f$-module $f E$ form a pair over $f R f$. Specifically, if $f_{1}, \cdots, f_{n}$ is a basic set of idempotents for $f$ and

$$
E=\oplus \sum E_{i j} \text { with } E_{i j} \cong E\left(T\left(R f_{i}\right)\right), \quad i=1, \cdots, n,
$$

then

$$
f E=\oplus \sum f E_{i j} \text { with } f E_{i j} \cong E\left(T\left(f R f_{i}\right)\right), \quad i=1, \cdots, n
$$

over $f R f$.

Proof. First we show that ${ }_{f R f} f E$ is injective. Every left ideal in the ring $f R f$ is of the form $f I f$ where If is a left ideal in $R$. If $\delta: f I f \rightarrow f E$ then by (2.1) $\delta$ can be extended to an $R$-map $\bar{\delta}: I f \rightarrow E$. Since $E$ is injective there is an $x$ in $E$ with $\bar{\delta}(a)=a x$ for all $a \in I f$. Thus $\delta(f a f)=f a f \cdot f x$ for all $f a f \in f I f$ and $f E$ is injective over $f R f$. To complete the proof we let $E_{i j} \cong E\left(T\left(R f_{i}\right)\right), f_{i}$ in a basic set of idempotents for $f$, and show that $S\left(f E_{i j}\right) \cong T\left(f R f_{i}\right)$ is simple over $f R f$. Since $S\left(E_{i j}\right)$ is simple and $f S\left(E_{i j}\right) \supseteq f_{i} S\left(E_{i j}\right) \neq 0$ it follows at once that $f S\left(E_{i j}\right)$ is simple and isomorphic to $T\left(f R f_{i}\right)$. Moreover, using $(1.1$, a),

$$
S\left(f E_{i j}\right) \subseteq \boldsymbol{r}_{E}(f N) \cap E_{i j}=\boldsymbol{r}_{E}(N) \cap E_{i j}=S\left(E_{i j}\right)
$$

so that

$$
f S\left(E_{i j}\right) \leqq S\left(f E_{i j}\right) \leqq f S\left(E_{i j}\right)
$$

This completes the proof. 
REMARK. The isomorphism $\rho$ in the proof of $(2.1, b)$ was pointed out by Rosenberg and Zelinsky in [15]. There they proved that if ${ }_{R} T$ is simple then $E(T)$ has finite length if and only if each of the left $R$-modules

$$
\operatorname{Hom}_{R}\left(N^{k-1} / N^{k}, T\right), \quad k=1,2, \cdots, m
$$

has finite length. Using a similar argument along with $(2.1, b)$ and (2.2) one can show that if $f$ is a primitive idempotent in $R$ then $E(T(R f))$ has finite length if and only if each of the left $R$-modules $\operatorname{Hom}_{f R f}\left(f N^{k-1} / f N^{k}, T(f R f)\right), k=1,2, \cdots$, has finite length. Thus the finiteness of an indecomposable injective left module $E$ over a semiprimary ring $R$ depends only upon the indecomposable projective right module $f R$, where $f$ is a primitive idempotent with $T(R f) \cong S(E)$.

Now we turn to left artinian rings. Our next lemma depends upon Tachikawa's generalized notion of orthogonal pairings.

Lemma 2.3. Let $R$ be a left artinian ring. Suppose $E$ is paired to $f R$ over $R$ and $S$ is the inverse $R$-endomorphism ring of $E$. Then

(a) ${ }_{R} E_{S} \cong \operatorname{Hom}_{f R f}(f R, f E),{ }_{f R f} f R_{R} \cong \operatorname{Hom}_{S}(E, f E)$;

(b) $\boldsymbol{l}_{f R}\left(\boldsymbol{r}_{E}(f J)\right)=f J, \boldsymbol{r}_{E}\left(\boldsymbol{l}_{f R}(M)\right)=M$, for each left ideal $J$ in $R$ and each right $S$-submodule $M$ of $E$.

Proof. In Tachikawa's terminology [16], it follows from $(1.1, b)$ that the left $f R f$-module $f R$ and the right $S$-module $E$ form an orthogonal pair with respect to $f E$ via the left $f R f$-right $S$-bilinear map

$$
(f r, x) \rightarrow f r x, f r \in f R, x \in E .
$$

Moreover, since $S$ may be regarded as the inverse $f R f$-endomorphism ring of $f E$ by (2.1) and $f E$ is an injective module over the left artinian ring $f R f$ whose socle is finitely generated and faithful over $f R f / f N f$ by (2.2), it follows from [16, Th. (2.1)] that $\operatorname{Hom}(T, f E)$ is simple for each simple left $f R f$-module and each simple right $S$-module. Now the left $f R f$-isomorphism and the right $S$-isomorphism of (a) and the equalities of (b) are consequences of [16, Th. (1.1)]. To complete the proof one checks that the right $S$-isomorphism $\rho: E \rightarrow \operatorname{Hom}(f R, f E)$ defined via

$$
[\rho(x)](f r)=f r x, x \in E, f r \in f R,
$$

and the left $f R f$ isomorphism $\lambda: f R \rightarrow \operatorname{Hom}(E, f E)$ defined via

$$
[\lambda(f r)](x)=f r x, f r \in f R, x \in E,
$$

are also $R$-maps. 
Our next result gives a natural one-to-one correspondence between the homogenious components of the $k$-th upper (lower) Loewy factor of $E$ and the $k$-th lower (upper) Loewy factor of $f R$ whenever $f R$ and $E$ form a pair over a left artinian ring $R$. We state the theorem in terms of indecomposable injectives and projectives.

THEOREM 2.4. Let $E$ be an indecomposable injective left module over a left artinian ring $R$. Left $f$ be a primitive idempotent in $R$ with $T(R f) \cong S(E)$. If $e$ is any primitive idempotent in $R$ then $T(R e)$ appears in the k-th upper (lower) Loewy factor of $E$ if and only if $T(e R)$ appears in the k-th lower (upper) Loewy factor of $f R$.

Proof. First we show that if $J^{\prime} \subseteq J$ are two-sided ideals in $R$ then

$$
\boldsymbol{l}_{R}\left(\boldsymbol{r}_{E}\left(J^{\prime}\right) / \boldsymbol{r}_{E}(J)\right)=\boldsymbol{r}_{R}\left(f J / f J^{\prime}\right) \text {. }
$$

It follows at once from $(2.1, \mathrm{~b})$ that $\boldsymbol{r}_{R}\left(f J / f J^{\prime}\right) \subseteq \boldsymbol{l}_{R}\left(\boldsymbol{r}_{E}\left(J^{\prime}\right) / \boldsymbol{r}_{E}(J)\right)$. For the reverse inclusion suppose that $f J r \nsubseteq f J^{\prime}, r \in R$. Then $\left(f J r+f J^{\prime}\right) / f J^{\prime}$ has a simple $f R f$-factor and using (2.2) we see that there is a map $\alpha \in \operatorname{Hom}_{f R f}\left(f J / f J^{\prime}, f E\right)$ such that $(r \alpha)\left(f J / f J^{\prime}\right)=\alpha\left(\left(f J r+f J^{\prime}\right) / f J^{\prime}\right) \neq 0$. Thus, by $(2.1, b), r$ does not annihilate $\boldsymbol{r}_{E}\left(J^{\prime}\right) / \boldsymbol{r}_{E}(J)$ and the desired equality holds.

Now taking $J=N^{k-1} \supseteqq N^{k}=J^{\prime}$ we see that if $e$ is a primitive idempotent in $R$ then $T(R e)$ can be embedded in $\boldsymbol{r}_{E}\left(N^{k}\right) / \boldsymbol{r}_{E}\left(N^{k-1}\right)$ if and only if $T(e R)$ can be embedded in $f N^{k-1} / f N^{k}$.

On the other hand, by $(2.3)$ and $(1.1, a)$,

$$
\begin{aligned}
N^{k} E & =\boldsymbol{r}_{E}\left(\boldsymbol{l}_{f R}\left(N^{k} E\right)\right)=\boldsymbol{r}_{E}\left(\boldsymbol{l}_{f R}\left(N^{k}\right)\right) \\
& =\boldsymbol{r}_{E}\left(f \boldsymbol{l}_{R}\left(N^{k}\right)\right)=\boldsymbol{r}_{E}\left(\boldsymbol{l}_{R}\left(N^{k}\right)\right) .
\end{aligned}
$$

So that, letting $J=\boldsymbol{l}_{R}\left(N^{k}\right) \supseteqq \boldsymbol{l}_{R}\left(N^{k-1}\right)=J^{\prime}$,

$$
\begin{aligned}
\boldsymbol{l}_{R}\left(N^{k-1} E / N^{k} E\right) & =\boldsymbol{l}_{R}\left(\boldsymbol{r}_{E}\left(\boldsymbol{l}_{R}\left(N^{k-1}\right)\right) / \boldsymbol{r}_{E}\left(\boldsymbol{l}_{R}\left(N^{k}\right)\right)\right) \\
& =\boldsymbol{r}_{R}\left(f \boldsymbol{l}_{R}\left(N^{k}\right) / f \boldsymbol{l}_{R}\left(N^{k-1}\right)\right)
\end{aligned}
$$

and the theorem is proved.

If $M$ is a left module over a semi-primary ring $R$ there is an indexed set of primitive idempotents $\left\{e_{\alpha} \mid \alpha \in I\right\}$ in $R$ such that

$$
T(M) \cong \bigoplus \sum_{\alpha \in I} T\left(R e_{\alpha}\right) \text {. }
$$

The projective cover of $M$ is isomorphic to $\oplus \sum_{\alpha \in I} R e_{\alpha}$ (see [2]). If $R$ is left artinian, $f$ is a primitive idempotent in $R$ and $E=E(T(R f))$ then it follows from (2.4) that the simple submodules of $T(E)$ (the 1-st upper Loewy factor of $E$ ) are precisely those of the form $T(R e)$ 
where $e$ is a primitive idempotent with $T(e R)$ isomorphic to a submodule of $S(f R)$ (the 1 st lower Loewy factor of $f R$ ). Thus we have

CoRollary 2.5. Let $E$ be an indecomposable injective left module over a left artinian ring $R$. Let $f$ be a primitive idempotent in $R$ with $T(R f) \cong S(E)$. If $e$ is any primitive idempotent in $R$ then $R e$ is isomorphic to a direct summand of the projective cover of $E$ if and only if $T(e R)$ is isomorphic to a submodule of $f R$.

A left artinian ring $R$ is $Q F-3$ in case $E\left({ }_{R} R\right)$ is projective or, equivalently, $R$ has a faithful injective projective left module. Using well known properties of injective and projective modules over a left artinian ring one can easily show that such a ring is $Q F-3$ if and only if the injective hull of each of its projective left modules is itself projective.

THEOREM 2.6. A left artinian ring is $Q F-3$ if and only if each of its injective left modules has an injective projective cover.

Proof. $(\Rightarrow)$ If ${ }_{R} E$ is an injective module over a left artinian ring $R$ then every simple submodule of $T(E)$ must be isomorphic to a simple factor of an indecomposable (injective) direct summand of $E$. Thus the projective cover of $E$ can be written $P=\oplus \sum R e_{\alpha}$ where, by (2.5), each $T\left(e_{\alpha} R\right)$ is isomorphic to a minimal right ideal in $R$. If $R$ is $Q F-3$ then according to [5, Th. (2.1)] each such $R e_{\alpha}$ is injective and hence so is $P$. (see [11, Lemma 3.5]).

$(\Leftarrow$ Let $R$ be a left artinian ring satisfying the condition. Let $e_{1}, \cdots, e_{m}$ be an orthogonal set of primitive idempotents such that $T\left(e_{1} R\right), \cdots, T\left(e_{m} R\right)$ represent the minimal right ideals in $R$. Let $f_{1}$, $\cdots, f_{m}$ be primitive idempotents such that $T\left(e_{i} R\right)$ is isomorphic to a submodule of $f_{i} R, i=1, \cdots, m$. Then by (2.5) $R e_{i}$ is isomorphic to a direct summand of the projective cover of $E\left(T\left(R f_{i}\right)\right), i=1, \cdots, m$, and by hypothesis, each $R e_{i}$ is injective. Moreover no minimal right ideal annihilates $R e_{1}+\cdots+R e_{m}$. Thus $R e_{1}+\cdots+R e_{m}$ is a faithful injective projective left $R$-module and $R$ is $Q F-3$.

3. Injective projective modules. In [5] we characterized twosided artinian $Q F-3$ rings in terms of one-sided ideal structure and a certain duality. The first theorem of this section gives similar characterizations of injective projective modules over left artinian rings. As a consequence [5, Th. 3.6] is valid for left artinian rings.

THEOREM 3.1. If $e$ is an idempotent element in a left artinian ring $R$ then the following statements are equivalent: 
(a) Re is injective.

(b) For each $e_{i}$ in a basic set of idempotents for $e$ there is a primitive idempotent $f_{i}$ in $R$ such that

$$
S\left(R e_{i}\right) \cong T\left(R f_{i}\right) \text { and } S\left(f_{i} R\right) \cong T\left(e_{i} R\right) \text {. }
$$

(c) There exists an idempotent $f$ in $R$ such that

(i ) $l_{f R}(R e)=0=r_{R e}(f R)$;

(ii) The functors

$$
\operatorname{Hom}_{f R f}(\ldots, f R e) \text { and } \operatorname{Hom}_{e R e}(\ldots, f R e)
$$

define a duality between the category of finitely generated left $f R f$ modules and the category of finitely generated right eRe-modules.

Moreover, if $R e$ is injective then the $f_{i} R$ of (b) and the $f R$ of (c) are also injective.

Proof. (a) $\Rightarrow$ (b) Here we may assume that $e$ is a primitive idempotent. Then by assumption $R e$ is an indecomposable injective module and there is a primitive idempotent $f$ in $R$ with $R e \cong E(T(R f)$ ). Thus, since the indecomposable injective left module $R e$ is paired to $f R$ and we may take $e R e$ as the inverse $R$-endomorphism ring of $R e$, we have by $(2.3, a)$

$$
f R \cong \operatorname{Hom}_{e R e}(R e, f R e) .
$$

Next we see that (1) $e R e$ is inverse-isomorphic to the $f R f$ endomorphism ring of $f R e$ via the map $[\psi($ ere $)](f t e)=$ fte ere, $r, t \in R$, by $(2.1, a)$; (2) $f R e$ is injective as a left $f R f$-module and the unique simple left $f R f$-module is isomorphic to the socle of $f R e$, by (2.2); (3) $f R e$ is finitely generated over the left artinian ring $f R f$. According to [10, Th. 6.3] these three conditions insure that Hom (_, $f R e)$ defines a duality between the categories of finitely generated left $f R f$ modules and finitely generated right $e R e$-modules. In particular $f R e \cong \operatorname{Hom}_{f R f}(f R f, f R e)$ must be the unique indecomposable injective right $e R e$-module $E(T(e R e))$. Now, applying the right-left symmetric versions of $(2.2)$ and $(2.1, \mathrm{~b})$ (with $J^{\prime}=0, J=R$ ), we have

$$
f R e \cong E(T(e R)) e
$$

as right $e R e$-modules and

$$
E(T(e R)) \cong \operatorname{Hom}_{e R e}(R e, f R e) .
$$

Thus

$$
f R \cong E(T(e R))
$$

and the relationships of $(b)$ follow. 
(b) $\Rightarrow$ (c) If $e_{i}$ and $f_{i}, i=1, \cdots, n$, satisfy the condition (b) we may assume $f_{1}, \cdots, f_{n}$ are also pairwise orthogonal. Let $f=f_{1}+\cdots+f_{n}$. Then no minimal right ideal in $f R$ anihilates $e$ and no minimal left ideal in $R e$ annihilates $f$, so (i) holds. To show that (ii) also holds we note that $[5$, Lemma $(3.5, b)]$ can be shown to be valid for our present $e$ and $f$ by replacing an argument involving the length of a composition series with one involving the length of a Loewy series. The implication now follows from reasoning identical to that of the proof that $(b) \Rightarrow$ (c) in [5, Th. (3.6)].

(c) $\Rightarrow$ (a) Let $e$ and $f$ satisfy the conditions of (c) and note that according to [10, Th. (6.3)] $e R e$ is right artinian. Write

$$
()^{*}=\operatorname{Hom}(\ldots, f R e) \text {. }
$$

Using (i) we see that if

$$
[\rho(r e)](f t)=f t r e, r e \in R e, f t \in f R
$$

and

$$
\mid \lambda(f r)](t e)=\text { frte }, f r \in f R, t e \in R e
$$

then $\rho$ is a left $R$-right $e R e$-monomorphism

$$
0 \longrightarrow R e \stackrel{\rho}{\longrightarrow}(f R)^{*},
$$

$R e$ is finitely generated over $e R e$, and $\lambda$ is a right $R$-left $f R f$-monomorphism

$$
0 \longrightarrow f R \stackrel{\lambda}{\longrightarrow}(R \mathrm{e})^{*} \text {. }
$$

Since a duality preserves composition lengths it follows that $\rho$ and $\lambda$ must be isomorphisms. In particular

$$
R e \cong \operatorname{Hom}_{f R f}(f R, f R e) \text {. }
$$

According to $[10$, Th. $(6.3, \mathrm{VI})]$ we can write

$$
{ }_{f R f} f R e \cong \bigoplus \sum \hat{E}_{i j}, \hat{E}_{i j} \cong E\left(T\left(f R f_{i}\right)\right), \quad i=1, \cdots, n,
$$

where $f_{1}, \cdots, f_{n}$ is a basic set of idempotents for $f$. Thus, by (2.2), if

$$
E=\oplus \sum E_{i j}, E_{i j}=E\left(T\left(R f_{i}\right)\right), \quad i=1, \cdots, n,
$$

we have $E$ paired to $f R$ and $f R e \cong f E$ over $f R f$. Now applying $\left(2.1\right.$, b) (with $J^{\prime}=0, J=R$ ) or $(2.3$, a) it follows that

$$
R e \cong \operatorname{Hom}_{f R f}(f R, f R e) \cong E
$$

is injective. 
In proving $(a) \Rightarrow(b)$ we showed that each $f_{i} R$ of $(b)$ is injective. A proof symmetric to that of (c) $\Rightarrow$ (a) shows that the $f R$ of (c) is injective.

COROLlARY 3.2. The indecomposable injective projective left modules over a left artinian ring $R$ are (to within isomorphism) precisely those modules of the form Re where $e$ is a primitive idempotent with

$$
S(R e) \cong T(R f) \text { and } S(f R) \cong T(e R)
$$

for some primitive idempotent $f$ in $R$.

COROLLARY 3.3. If $R$ is a ring with minimum conditions on left and right ideals there is a natural one-to-one correspondence between the sets of isomorphism classes of indecomposable injective projective left $R$-modules and indecomposable injective projective right $R$-modules.

The characterizations of injective projective modules given in (3.1) clearly generalize Nakayama's definition of $Q F$ rings as well as the characterization of these rings in terms of duality. Our next result generalizes the annihilator characterization of $Q F$-rings.

THEOREM 3.4. Let $e$ be an idempotent element in a left artinian ring $R$. Then $R e$ is injective if and only if there is an idempotent $f$ in $R$ such that

$$
\boldsymbol{l}_{f R}\left(\boldsymbol{r}_{R e}(I)\right)=f I \text { and } \boldsymbol{r}_{R e}\left(\boldsymbol{l}_{f R}(J)\right)=J e
$$

for each left ideal $I$ and each right ideal $J$ of $R$.

Proof. $(\Rightarrow)$ If $R e$ is injective, take $f \in R$ such that $f R$ and $R e$ form a pair. Then by (1.1) $\boldsymbol{r}_{R e}(I)=\boldsymbol{r}_{R e}(f I)$ and $\boldsymbol{l}_{f R}(J)=\boldsymbol{l}_{f R}(J R e)=$ $\boldsymbol{l}_{f R}(J e)$. Also $J e$ is stable under $R$-endomorphisms of $R e$. Therefore the annihilator conditions hold by (2.3). Then

$(\Longleftrightarrow)$ Suppose $R$ e and $f R$ satisfy the given annihilator conditions.

$$
\boldsymbol{l}_{f R}(R e)=\boldsymbol{l}_{f R}\left(\boldsymbol{r}_{R e}(0)\right)=0=\boldsymbol{r}_{R e}\left(\boldsymbol{l}_{f R}(0)\right)=\boldsymbol{r}_{R e}(f R),
$$

so $R e$ and $f R$ satisfy part (i) of $(3.1, \mathrm{c})$. To show that part (ii) of $(3.1, \mathrm{c})$ holds we once again appeal to Morita's duality theorem [10, Th. (6.3)]. According to condition $\mathrm{V}$ of that theorem, we need only show that (1) for every left ideal $I$ of $f R f$ and every right $e R e$-submodule $W$ of $f R e$,

$$
I=\boldsymbol{l}_{f R f}\left(\boldsymbol{r}_{f R e}(I)\right) \text { and } W=r_{f R e}\left(\boldsymbol{l}_{f R f}(W)\right)
$$


and (2) for every left $f R f$-submodule $V$ of $f R e$ and every right ideal $J$ of $e R e$,

$$
V=\boldsymbol{l}_{f R e}\left(\boldsymbol{r}_{e R e}(V)\right) \text { and } J=\boldsymbol{r}_{e R e}\left(\boldsymbol{l}_{f R e}(J)\right) \text {. }
$$

If $I$ is a left ideal of $f R f$ then $I=f I f$ and

$$
\boldsymbol{r}_{R e}(I) \subseteq f \boldsymbol{r}_{R e}(I)+(1-f) \boldsymbol{r}_{R e}(I) \subseteq \boldsymbol{r}_{f R e}(I)+(1-f) R e .
$$

Thus we have

$$
\begin{aligned}
I=f R I & =\boldsymbol{l}_{f R}\left(\boldsymbol{r}_{R e}(R I)\right) \supseteqq \boldsymbol{l}_{f R}\left(\boldsymbol{r}_{R e}(I)\right) \supseteqq \boldsymbol{l}_{f R f}\left(\boldsymbol{r}_{R e}(I)\right) \\
& \supseteqq \boldsymbol{l}_{f R f}\left(\boldsymbol{r}_{R e}(I)+(1-f) R e\right) \\
& \left.=\boldsymbol{l}_{f R f}\left(\boldsymbol{r}_{f R e}(I)\right) \cap \boldsymbol{l}_{f R f}(1-f) R e\right)=\boldsymbol{l}_{f R f}\left(\boldsymbol{r}_{f R e}(I)\right) .
\end{aligned}
$$

Since it is obvious that $I \subseteq \boldsymbol{l}_{f R f}\left(\boldsymbol{r}_{f R e}(I)\right)$, we now have the first part of (1). The proof of the rest of (a) is similar. By a symmetric argument (2) also holds and the theorem is proved.

Note the conditions (b) and (c) of (3.1) and the condition of (3.4) give characterizations of left artinian $Q F-3$ rings if we demand that $R e$ be faithful. Also as a consequence of (3.1) we have a generalization of a theorem that Harada [6] proved for rings with both minimum conditions.

Theorem 3.5. Let $R$ be a left artinian $Q F-3$ ring. Then $E\left(R_{R}\right)$ is projective and $S\left(R_{R}\right)$ has finite composition length.

Proof. Let $e$ be an idempotent in $R$ with $R e$ a faithful injective projective left $R$-module. Let $f$ be an idempotent in $R$ such that $f R$ and $R e$ form a pair. Then by (3.1) $f R$ is injective and by (1.1, c) $f R$ is faithful. Now once we see that $S\left(R_{R}\right)$ has finite composition length we will have that $E\left(R_{R}\right)$ is a direct summand of a direct sum of a finite number of copies of $f R$ (since faithful right modules contain copies of all minimal right ideals) and hence is projective. To this end, note that no minimal right ideal of $R$ annihilates $R e$ so that if $S\left(R_{R}\right)=\oplus \sum_{\alpha \in I} S_{\alpha}, S_{\alpha}$ simple, then $S\left(R_{R}\right) e=\bigoplus \sum_{\alpha \in I} S_{\alpha} e, S_{\alpha} e$ simple over $e R e$. Thus $S\left(R_{R}\right)$ has finite length over $R$ if $S\left(R_{R}\right) e$ has finite length over $e R e$, and the latter is true by (3.4).

The question arises whether every left artinian $Q F-3$ ring is necessarily right artinian. Theorem (3.5) reduces this question to one of the finiteness of the indecomposable injective projective right modules over such a ring. Moreover, we note that if each factor ring of a left artinian ring is $Q F-3$ then it follows from (3.5) that $R$ is also right artinian. Independently Morita [10] and Wall [18] proved 
that this condition is necessary and sufficient for a finite dimensional algebra to be a generalized uniserial ring. Morita's proof [10, Th. (17.8)] is valid for two-sided artinian rings. Thus we see that

Theorem 3.6. A left artinian ring is generalized uniserial if and only if each of its factor rings is $Q F-3$.

4. Indecomposable quasi-injectives. An $R$-module $M$ is quasiinjective in case every $\mathrm{R}$-map of a submodule of $M$ into $M$ can be extended to an $R$-endomorphism of $M$ (see [9].) Wu and Jans [19] have recently considered the dual notion-an $R$-module $L$ is quasiprojective in case every $R$-map of $L$ into a factor module of $L$ is the composite of an $R$-endomorphism of $L$ and the natural epimorphism.

Let $R$ be a semi-primary ring. Then it follows from [9, Th. (1.1)] and an argument given in [19, Proposition (2.4)] that

Theorem. A left $R$-module $M$ is an indecomposable quasi-injective module if and only if $E(M)$ is indecomposable and $M$ is stable under $R$-endomorphisms of $E(M)$.

$\mathrm{Wu}$ and Jans [19] proved the following theorem that is valid in the same setting.

THeOREM. A left $R$-module $L$ is an indecomposable quasi-projective module if and only if

$$
L \cong R e / J e
$$

where $e$ is a primitive idempotent and $J$ is a two-sided ideal in $R$.

These results leads us to consider how the duality relations of $\S 2$ apply to indecomposable quasi-injective and quasi-projective modules.

THEOREM 4.1. Let $f$ be a primitive idempotent in a left artinian ring $R$. Let $E=E(T(R f)$ ) and let $S$ be the inverse $R$-endomorphism ring of $E$. Then

and

$$
X \rightarrow f R / l_{f R}(X), X \leqq{ }_{R} E_{S}
$$

$$
f R / f J \rightarrow \boldsymbol{r}_{E}(J), J \leqq{ }_{R} R_{R}
$$

provide inverse bijections between the set of quasi-injective submodules of $E$ and the set of quasi-projective factors of $f R$.

Proof. Using the two theorems cited above one can quickly check 
that the given associations define mappings between the desired sets. They are bijections because, using $(2.3, b)$ and $(1.1, a)$,

$$
\boldsymbol{r}_{E}\left(\boldsymbol{l}_{f R}(X)\right)=X
$$

and

$$
\boldsymbol{l}_{f R}\left(\boldsymbol{r}_{E}(J)\right)=\boldsymbol{l}_{f R}\left(\boldsymbol{r}_{E}(f J)\right)=f J .
$$

The fact that $f R / f J \rightarrow r_{E}(J)$ is surjective gives us the dual to the Wu-Jans characterization of indecomposable quasi-projectives.

CoRollaRy 4.2. If $R$ is a left artinian ring, a left $R$-module $M$ is an indecomposable quasi-injective module if and only if

$$
M \cong \boldsymbol{r}_{E}(J)
$$

where $E$ is an indecomposable injective left $R$-module and $J$ is a two-sided ideal in $R$.

If $R$ is right artinian with two-sided ideal $J$ and $J^{\prime}$ and a primitive idempotent $f$ then it follows from an argument given in $[19, \mathrm{p}$. 447] that $f J=f J^{\prime}$ whenever $f R / f J \cong f R / f J^{\prime}$. On the other hand if $X$ and $X^{\prime}$ are isomorphic quasi-injective submodules of $E=E(T(R f))$ it is easy to show that, since $X$ and $X^{\prime}$ are stable under endomophisms of $E, X=X^{\prime}$. Thus we have

CoRollary 4.3. Let $R$ be a ring with both minimum conditions. If $M$ is an $R$-module let $[M]$ be the isomorphism class of $M$. Then

$$
[X] \leftrightarrow\left[f R / l_{f R}(X)\right], S(X) \cong T(R f)
$$

defines a one-to-one correspondence between the isomorphism classes of indecomposable left quasi-injectives and the isomorphism classes of indecomposable right quasi-projectives over $R$.

We note that the above correspondence can be shown to be precisely that given by the vector space dual if $R$ is a finite dimensional algebra.

The next theorem shows that the indecomposable quasi-injective modules over $R$ are nothing more nor less than the indecomposable injective modules over the factor rings of $R$.

THEOREM 4.4. Let $M$ be an indecomposable left module over a left artinian ring $R$. Then the following are equivalent.

(a) $M$ is quasi-injective.

(b) $M$ is injective over $R / J$ for some two-sided ideal $J \subseteq l_{R}(M)$.

(c) $M$ is injective over $R / l_{R}(M)$. 
Proof. $\quad(b) \Leftrightarrow$ (c) We omit this easy proof.

(c) $\Rightarrow$ (a) Suppose $M$ is an $R$-module injective over $R / l_{R}(M)$ and $L \leqq{ }_{R} M$ with $\alpha: L \rightarrow M$ over $R$. Then $\alpha ; L \rightarrow M$ over $R / l_{R}(M)$ and $\alpha$ can be extended to an endomorphism of $M$.

(a) $\Rightarrow$ (b) This implication follows from (4.2) and the next lemma.

LEMMA 4.5. Let $f$ be a primitive idempotent in a simi-primary ring $R$. Let $E=E(T(R f)$ ) and suppose $J$ is a two-sided ideal in $R$ with $f \notin J$. Then $T(R f)$ is a simple $R / J$-module and

$$
E\left({ }_{R / J} T(R f)\right) \cong r_{E}(J) .
$$

Proof. If $J T(R f) \neq 0$ then $J f \nsubseteq N f$, so $J f=R f$ and $f \in J$. Thus by assumption $J T(R f)=0$ and $T(R f)$ is an $R / J$-module. Since any of its $R / J$-submodules must be $R$ submodules, $T(R f)$ is simple over $R / J$. Also $T(R f) \subseteq \boldsymbol{r}_{E}(J)$ so $\boldsymbol{r}_{E}(J)$ is an essential extension of $T(R f)$. Suppose $L \leqq{ }_{R / J} M$ and $\alpha: L \rightarrow \boldsymbol{r}_{E}(J)$ over $R / J$. Then we can regard $\alpha$ as an $R$-map $\alpha: L \rightarrow E$, so there is an $R$-map $\bar{\alpha}: M \rightarrow E$ that extends $\alpha$. But $J \bar{\alpha}(M)=\bar{\alpha}(J M)=0$ and we really have $\bar{\alpha}: M \rightarrow \boldsymbol{r}_{E}(J)$. This proves the lemma.

Wu and Jans [19] showed that a left artinian ring has an infinite number of non-isomorphic indecomposable left quasi-projectives if and only if it has an infinite ideal lattice. Dually

THEOREM 4.6. A left artinian ring has an infinite number of nonisomorphic indecomposable quasi-injective left modules if and only if it has an infinite lattice of two sided ideals.

Proof. $(\Longrightarrow)$ If the lattice of two-sided ideals of a left artinian ring $R$ is finite then $R$ has only a finite number of left indecomposable quasi-injectives by (4.4).

$(\Longleftrightarrow$ If $R$ has an infinite lattice of two-sided ideals, then, writing $1 \in R$ as a sum of primitive orthogonal idempotents, we see that there is a primitive right ideal $f R$ with an infinite number of distinct submodules of the form $f J, J$ a two-sided ideal in $R$. Thus $E(T(R f))$ must have an infinite number of distinct quasi-injective submodules by (4.1). As we pointed out in the comments preceeding (4.3), no two of these are isomorphic.

5. Quasi-projective quasi-injectives. In $\S 4$ we saw that the correspondence ${ }_{R} X \rightarrow f R / l_{f R}(X), T(R f) \cong S(X)$ takes indecomposable quasi-injectives to indecomposable quasi-projectives over a left artinian ring $R$. The following theorem shows that this correspondence also 
takes quasi-projective quasi-injectives to quasi-injective quasi-projectives.

THEOREM 5.1. Let $R$ be a left artinian ring with primitive idempotents $e$ and $f$. Suppose there exist two-sided ideals $J$ and $K$ in $R$ such that

$$
\boldsymbol{r}_{E(T(R f))}(J) \cong R e / K e
$$

Then

$$
f R / f J \cong l_{E(T(e R))}(K)
$$

Proof. Throughout this proof we shall use (1.1), its right-left dual dual version and (2.3) without reference. Let $e$ and $f$ be primitive idempotents in $R$, let $E=E(T(R f))$ and $E^{\prime}=E(T(e R))$, and suppose

$$
r_{E}(J) \cong R e / K e
$$

for some two-sided ideals $J$ and $K$ in $R$. Then we must have $J \cdot R e / K e=0$ and $K \cdot \boldsymbol{r}_{E}(J)=0$ so that $J e=J R e \leqq K e$ and $f K \cong l_{f R}\left(\boldsymbol{r}_{E}(J)\right)=$ $\boldsymbol{l}_{f R}\left(\boldsymbol{r}_{E}(f J)\right)=f J$. Thus

$$
(J+K) e=K e \text { and } f(J+K)=f J .
$$

Moreover we have

$$
\boldsymbol{r}_{E}(J+K)=\boldsymbol{r}_{E}(f(J+K))=\boldsymbol{r}_{E}(f J)=\boldsymbol{r}_{E}(J)
$$

and

$$
l_{E^{\prime}}(J+K)=l_{E^{\prime}}((J+K) e)=l_{E^{\prime}}(K e)=l_{E^{\prime}}(K) .
$$

If $e \in J+K$ then $R e \subseteq(J+K) e=K e, \boldsymbol{r}_{E}(f J)=\boldsymbol{r}_{E}(J)=0$ and $f J=$ $\boldsymbol{l}_{f R}\left(\boldsymbol{r}_{E}(f J)\right)=\boldsymbol{l}_{f R}(0)=f R$. So that

$$
f R / f J \cong 0=l_{E^{\prime}}(R e)=l_{E^{\prime}}(K e)=l_{E^{\prime}}(K) .
$$

If $f \in J+K$ then $f R \cong f(J+K)=f J, \boldsymbol{r}_{E}(J)=\boldsymbol{r}_{E}(f J)=\boldsymbol{r}_{E}(f R)=0$ and $R e=K e$. So again

$$
f R / f J \cong 0=l_{E^{\prime}}(K) .
$$

If neither $e$ nor $f$ is contained in $J+K$ let

$$
\bar{R}=R /(J+K), \bar{e}=e+(J+K), \bar{f}=f+(J+K) .
$$

Then as $\bar{R}$-modules

$$
\bar{R} \bar{e} \cong R e / K e, \bar{f} \bar{R} \cong f R / f J
$$


and, by (4.5) and its right-left symmetric version,

$$
E(T(\bar{R} \bar{f})) \cong r_{E}(J), E(T(\bar{e} \bar{R})) \cong \boldsymbol{l}_{E^{\prime}}(K) \text {. }
$$

But now we have, over $\bar{R}$.

$$
\bar{R} \bar{e} \cong E(T(\bar{R} \bar{f})) .
$$

So, according to (3.1), $S(\bar{f} \bar{R}) \cong T(\bar{e} \bar{R})$ and $\bar{f} \bar{R}$ is injective over $\bar{R}$. That is, as $\bar{R}$ modules

$$
\bar{f} \bar{R} \cong E(T(\bar{e} \bar{R}))
$$

Therefore

$$
f R / f J \cong \boldsymbol{l}_{E}(K)
$$

over $R$.

CoRollary 5.2. Let $R$ have both minimum conditions. In the one-to-one correspondence between indecomposable left quasi-injectives and indecomposable right quasi-projectives

$$
[X] \leftrightarrow\left[f R / l_{f R}(X)\right], T(R f) \cong S(X),
$$

$X$ is quasi-projective (projective) if and only if $f R / l_{f R}(X)$ is quasiinjective (injective).

Proof. If $R$ is both left and right artinian than (5.1) and its converse are true. Thus a left indecomposable quasi-injective $X$ with $S(X) \cong T(R f)$ is quasi-projective if and only if $X \cong R e / K e, e$ primitive, $K$ an ideal, if and only if $f R / \boldsymbol{l}_{f R}(X) \cong \boldsymbol{l}_{\left.E\left(T^{(} e R\right)\right)}(K), e$ primitive, $K$ an ideal, if and only if $f R / \boldsymbol{l}_{f R}(X)$ is quasi-injective. To see that $X$ is projective if and only if $f R / \boldsymbol{l}_{f R}(X)$ is injective take $K=0$.

In conclusion we apply (5.1) to get several characterizations of generalized uniserial rings. Let us say that a module over an artinian ring $R$ is uniserial in case it has a unique composition series of submodules. One can easily check that an $R$-module is uniserial if and only if each of its nontrivial upper (equivalently, lower) Loewy factors is simple. Thus the upper (lower) Loewy series for a uniserial module is its unique composition series. The ring $R$ is generalized uniserial in case each of its indecomposable projective modules is uniserial. One can show directly that generalized uniserial rings satisfy each of the conditions in the next two theorems by using Nakayama's theorem [13, Th. 17] that each indecomposable module over a generalized uniserial ring is an epimorph of an indecomposable projective.

THEOREM 5.3. Let $R$ be a left artinian ring. Then the following are equivalent. 
(a) $R$ is generalized uniserial.

(b) Every indecomposable $R$-module is quasi-projective.

(c) Every indecomposable $R$-module is quasi-injective.

(d) Every indecomposable quasi-injective $R$-module is quasiprojective.

(e) Every indecomposable quasi-projective $R$-module is quasiinjective.

(a) $\Rightarrow$ (b) According to Nakayama [13, Th. 17] each indecomposable left (right) module $M$ over a generalized uniserial ring is isomorphic to $R e / N^{k} e\left(e R / e N^{k}\right)$ for some primitive idempotent $e$ and some nonnegative integer $k$. But such a module is quasi-projective according to [19].

(b) $\Rightarrow$ (d) Trivially.

(d) $\Rightarrow$ (e) Let $f$ be a primitive idempotent in $R$ and $J$ a two-sided ideal. Then by assumption and [19] the indecomposable quasi-injective left module $r_{E(T(R f))}(J) \cong R e / K e, e$ primitive, $K$ an ideal. Now applying (5.1) we have $f R / f J \cong \boldsymbol{l}_{E(T(e R))}(K)$ is quasi-injective. Thus each right indecomposable quasi-projective is quasi-injective over $R$. In particular the indecomposable quasi-injective module $f R / \boldsymbol{l}_{f R}\left(N^{k}\right)$ has a simple socle for each $k$ with $l_{f R}\left(N^{k}\right) \neq f R$. Thus $R$ is right artinian and, by symmetry, each indecomposable left quasi-projective is also quasi-injective.

(e) $\Longrightarrow$ (a) We noted above that if $f R / l_{f R}\left(N^{k}\right), f$ primitive, is quasiinjective then $\boldsymbol{l}_{f R}\left(N^{k+1}\right) / \boldsymbol{l}_{f R}\left(N^{k}\right)$ is simple or zero.

(b) $\Rightarrow$ (c) This proof is just like (d) $\Rightarrow$ (e).

(c) $\Rightarrow$ (e) Trivially.

As far as we know the next theorem and (3.6) provide the first characterizations of generalized uniserial rings in terms of the category of left modules over a ring.

THEOREM 5.4. The following statements about a left artinian ring $R$ are equivalent.

(a) $R$ is generalized uniserial.

(b) Every indecomposable left $R$-module is both quasi-projective and quasi-injective.

(c) Every indecomposable left $R$-module is uniserial.

(d) For each primitive idempotent $e$ in $R$, Re and $E(T(R e))$ are uniserial.

Proof. (a) $\Rightarrow$ (b) This is a consequence of (5.3).

(b) $\Rightarrow$ (c) See the proof of (e) $\Rightarrow$ (a) in (5.3).

(c) $\Rightarrow$ (d) Trivially. 
(d) $\Rightarrow$ (a) We shall show that if $f$ is a primitive idempotent in a ring $R$ that satisfies (d), $f R / f N^{k}, k \geqq 1$ is quasi-injective and hence has $f N^{k-1} / f N^{k}$ simple or zero. Let $E=E(T(R f))$. Then $T\left(r_{E}\left(N^{k}\right)\right)$ is simple so that $r_{E}\left(N^{k}\right)$ is an epimorph or $R e$ for some primitive idempotent $e$ in $R$. Since $R e$ is uniserial we must have $r_{E}\left(N^{k}\right) \cong R e / K e$ where $K$ is some power of $N$. Now by (5.1) $f R / f N^{k} \cong \boldsymbol{l}_{E(T(e R))}(K)$ is quasi-injective and the theorem is proved.

In closing we remark on the obvious question that arises from (5.4). Is an artinian ring $R$ necessarily generalized uniserial if each of its indecomposable injectives is uniserial? The answer is, of course, yes if $R$ is a finite dimensional algebra. However, the best we can say in the general case is that (2.4) implies that the Loewy factors of the indecomposable projectives over such a ring must be homogenious; and by (4.1) the only $f R f-R$-submodules of $f R, f$ primitive, are those of the form $f N^{k}, k=0,1, \cdots$.

Added in proof. G. Azumaya has a more general result than Lemma (4.5) in Theorem 17 of $A$ duality theory for injective modules, Amer. J. Math. 81 (1959), 249-278.

\section{BIBLIOGRAPHY}

1. E. Artin, C. J. Nesbitt and R. M. Thrall, Rings with minimum condition, University of Michigan Press, Ann Arbor, 1943.

2. H. Bass, Finitistic dimension and a homological generalization of semi-primary ring, Trans. Amer. Math. Soc. 95 (1960), 466-488.

3. B. Eckmann and A. Schopf, Uber injective Modulen, Archiv der Math. 4 (1953), 75-78.

4. S. Eilenberg, Homological dimension and syzygies, Ann. of Math. 64 (1956), 328336.

5. K. R. Fuller, The structure of $Q F-3$ rings, Trans. Amer. Math. Soc. 134 (1968), 343-354.

6. M. Harada, QF-3 and semi-primary PP-rings, II, Osaka J. Math. 3 (1966), 21-27.

7. N. Jacobson, Structure of Rings, Providence, R. I., 1964.

8. J. P. Jans, Projective injective modules, Pacific J. Math. 9 (1959),1103-1108.

9. R. E. Johnson and E. T. Wong, Quasi-injective modules and irreducible rings, J. London Math. Soc. 36 (1961), 260-268.

10. K. Morita, Duality of modules and its applications to the theory of rings with minimum condition, Sci. Rep. Tokyo Kyoiku Daigaku 6 (1958), 85-142.

11. K. Morita, Y. Kawada and H. Tachikawa, On injective modules, Math. Z., 68 (1957), 217-226.

12. H. Nagao and T. Nakayama, On the structure of $\left(M_{0}\right)$-and $\left(M_{u}\right)$-modules, Math. $\mathrm{Z}$.

59 (1953), 164-170.

13. T. Nakayama, On Frobeniusean algebras, II, Ann. of Math. 42 (1941), 1-22.

14. M. Osima, Notes on basic rings, I, Math. J. Okayama Univ. 2 (1953), 103-110.

15. A. Rosenberg and D. Zelinsky, Finiteness of the injective hull, Math. Z. 70 (1959), 372-380.

16. H. Tachikawa, Duality theorem of character modules for rings with minimum 
conditition, Math. Z. 68 (1958), 479-487.

17. R. M. Thrall, Some generalizations of quasi-Frobenius algebras, Trans. Amer. Math. Soc. 64 (1948), 173-183.

18. D. W. Wall, Characterizations of generalized uniserial algebras, II, Proc. Amer. Math. Soc. 9 (1958), 915-919.

19. L. E. T. Wu and J. P. Jans, On quasi projectives, Illinois J. Math, 11 (1967), 139-448.

Received June 5, 1968.

UNIVERSITY OF IOWA

IoWA CiTY, IowA 




\section{Pacific Journal of Mathematics \\ Vol. 29, No. 1 \\ May, 1969}

Jorge Alvarez de Araya, A Radon-Nikodým theorem for vector and operator

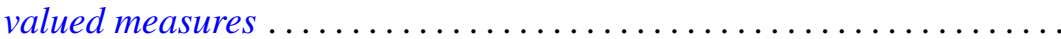

Deane Eugene Arganbright, The power-commutator structure of finite

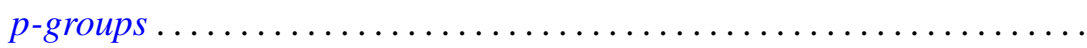

Richard Eugene Barlow, Albert W. Marshall and Frank Proschan, Some

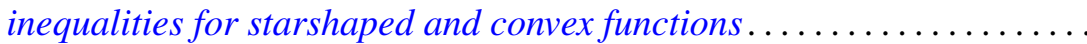

David Clarence Barnes, Some isoperimetric inequalities for the eigenvalues

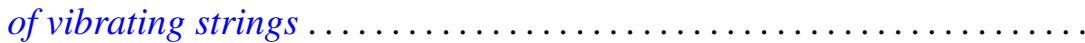

David Hilding Carlson, Critical points on rim-compact spaces ...........

Allan Matlock Weber Carstens, The lattice of pretopologies on an arbitrary

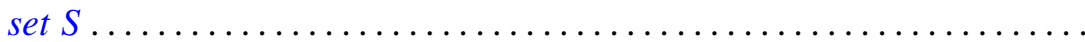

S. K. Chatterjea, A bilateral generating function for the ultraspherical

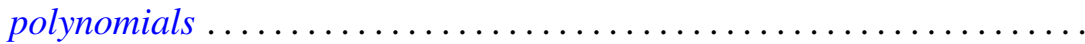

Ronald J. Ensey, Primary Abelian groups modulo finite groups ......... 77

Harley M. Flanders, Relations on minimal hypersurfaces ............ 83

Allen Roy Freedman, On asymptotic density in n-dimensions........... 95

Kent Ralph Fuller, On indecomposable injectives over artinian rings...... 115

George Isaac Glauberman, Normalizers of p-subgroups in finite groups . . . 137

William James Heinzer, On Krull overrings of an affine ring ........... 145

John McCormick Irwin and Takashi Ito, A quasi-decomposable abelian group without proper isomorphic quotient groups and proper isomorphic subgroups.

Allan Morton Krall, Boundary value problems with interior point boundary conditions

John S. Lowndes, Triple series equations involving Laguerre

polynomials

Philip Olin, Indefinability in the arithmetic isolic integers

Ki-Choul Oum, Bounds for the number of deficient values of entire functions whose zeros have angular densities..

R. D. Schafer, Standard algebras ....................

Wolfgang M. Schmidt, Irregularities of distribution. III.

Richard Alfred Tapia, An application of a Newton-like method to the Euler-Lagrange equation 